Case Report

\title{
A Case of Contagious Ecthyma (Orf Virus) in a Nonmanipulated Laboratory Dorset Sheep (Ovis aries)
}

\author{
Gwynne E. Kinley, Connie W. Schmitt, and Julie Stephens-Devalle \\ Veterinary Services Program, Walter Reed Army Institute of Research/Naval Medical Research Center, 511 Robert Grant Avenue, \\ Silver Spring, MD 20910, USA \\ Correspondence should be addressed to Julie Stephens-Devalle; j.stephensdevalle@us.army.mil
}

Received 25 April 2013; Accepted 23 June 2013

Academic Editors: L. G. Papazoglou and M. Pizarro

Copyright (C) 2013 Gwynne E. Kinley et al. This is an open access article distributed under the Creative Commons Attribution License, which permits unrestricted use, distribution, and reproduction in any medium, provided the original work is properly cited.

\begin{abstract}
An approximately 5-month-old laboratory wether, originating from a local vendor with a closed flock and maintained on a preventative medicine plan, presented with a continuum of lesions from hemorrhagic papules, vesicles, and pustules, to multifocal necrotic scabs at the commissure of the lips, medial canthus of the left eye, and distal prepuce. A presumptive diagnosis of Orf virus (ORFV) was made and the sheep was euthanized. A full necropsy was performed, and histopathological evaluation of affected tissues revealed multifocal-to-coalescing necrotizing and proliferative cheilitis and dermatitis with eosinophilic intracytoplasmic inclusion bodies. Electron microscopy findings revealed degenerate keratinocytes containing numerous typical 200-300 nm wide cytoplasmic parapoxvirus virions, confirming the diagnosis of ORFV. We believe that this animal developed a clinical case of ORFV either due to an adverse reaction to an ORFV vaccine, or this animal had a case of preexisting ORFV which manifested after arrival at our facility.
\end{abstract}

\section{Case History and Presentation}

On June 13, 2012, an approximately 5-month-old, $24 \mathrm{~kg}$, Dorset wether (Ovis aries) was introduced to our facility from a local vendor. Upon arrival, this animal was determined to be healthy based on history, general health, and physical exam. The health certificate that accompanied the animal indicated it had been examined and was clinically free of ORFV, keratoconjunctivitis, contagious foot-rot, and scabies. The WRAIR/ NMRC protocol requires all wethers to be sheared, docked, negative for Q fever and ORFV, and dewormed prior to shipment. This animal was acclimated in our vivarium. During the first four days of the acclimation period, the sheep displayed no abnormal behavioral or clinical signs. It demonstrated a healthy appetite and was fed ad libitum Rumilab diet 5508 (LabDiet supplier, Quality Lab Products, Elkridge, MD, USA), Timothy Hay (Kaytee Products, Inc., Chilton, WI, USA), and received water ad libitum. This Dorset sheep was pair-housed in a standard large animal aluminum run with polyvinyl chloride- (PVC-) coated steel mesh floor racks. The environment was maintained at 68 to 72 degrees $\mathrm{F}$, with a relative humidity range of 30 to $70 \%$ and a 12 :12-hour light: dark cycle. Environmental enrichment was provided in accordance with institute standard operating procedures. All animal handling and husbandry were performed by trained personnel, and the facility was maintained according to accepted animal care and use standards [1]. The sheep was procured for a protocol reviewed and approved by the WRAIR/NMRC Institutional Animal Care and Use Committee (IACUC). WRAIR/NMRC is fully accredited by the Association for the Assessment and Accreditation of Laboratory Animal Care International. Five days after its arrival at our vivarium, the sheep presented with a continuum of lesions from hemorrhagic papules, vesicles, and pustules to multifocal necrotic scabs at the commissure of the lips, medial canthus of the left eye, and distal prepuce. A presumptive diagnosis of ORFV was made, and the sheep was euthanized and submitted to the WRAIR/NMRC Department of Pathology (Silver Spring, MD, USA) to confirm ORFV.

\section{Analysis and Findings}

A complete necropsy was performed, and sections of the affected haired skin at the mucocutaneous junctions of the 


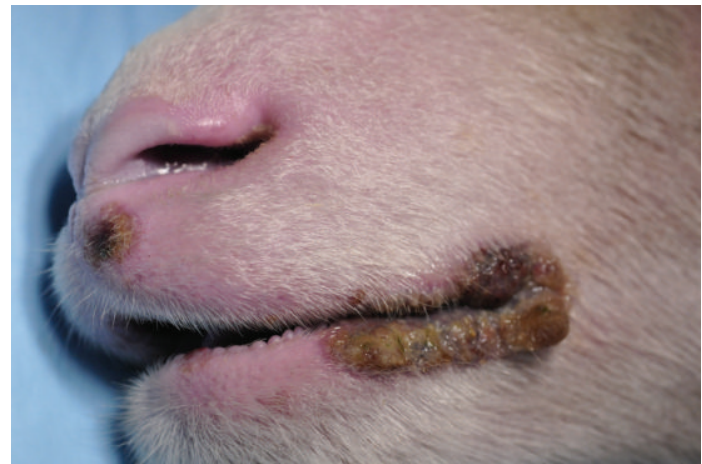

FIGURE 1: Left lips and philtrum. Multifocal-to-coalescing necrotizing and proliferative cheilitis and dermatitis.

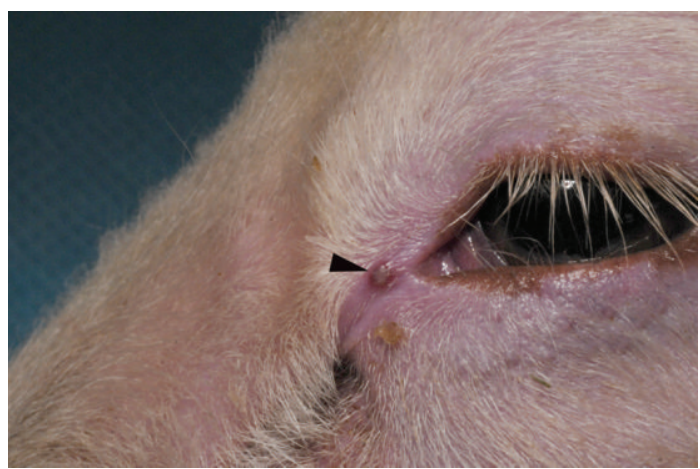

FIGURE 2: Left palpebrae, medial canthus. Focal hemorrhagic pustule (arrowhead).

lips, left eye, and prepuce were submitted for histopathological evaluation. Significant gross necropsy findings included scattered hemorrhagic papules, vesicles, pustules, and numerous multifocal-to-coalescing proliferative and necrotizing scabs affecting haired skin at the mucocutaneous junctions (commissures) of the lips, extending into and affecting the oral papillae, the medial canthus of the left eye, and the distal prepuce (Figures 1, 2, and 3). There were no other significant gross lesions to suggest an underlying immunocompromising condition.

All tissues for histologic assessment were fixed in 10\% neutral buffered formalin and routinely processed as $5 \mu \mathrm{m}$ thick paraffin sections and stained with hematoxylin and eosin stain (H\&E). The haired skin at the mucocutaneous junction of the lips (Figures 4, 5, and 6) revealed multifocal epithelial hyperplasia with increased thickness up to 10 times normal (acanthosis) and elongated, anastomosing rete ridges. Multifocally within the epithelium, there was prominent intracellular bridging (spongiosis), neutrophilic inflammation, and multifocal vesicles. Multifocally, the keratinocytes were swollen and often contained clear intracytoplasmic vacuoles (ballooning degeneration) and pyknotic nuclei. Rarely keratinocytes contained one or more 2-10 $\mu \mathrm{m}$ round, brightly eosinophilic intracytoplasmic inclusion bodies. Focally extensive, the epidermis exhibited erosion, loss and

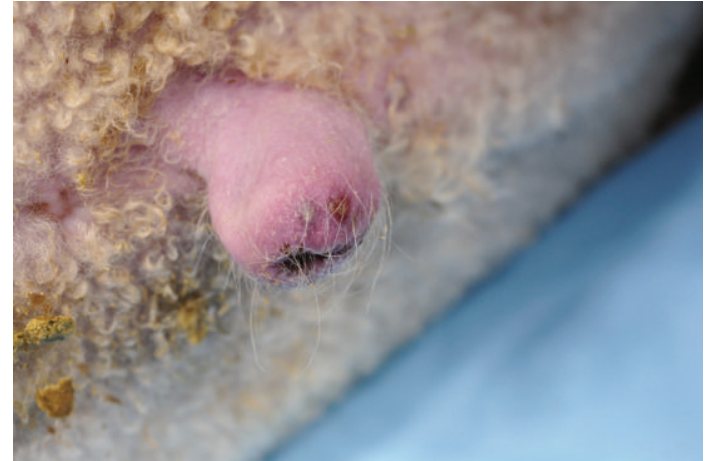

FIGURE 3: Prepuce. Multifocal necrotizing and proliferative posthitis.

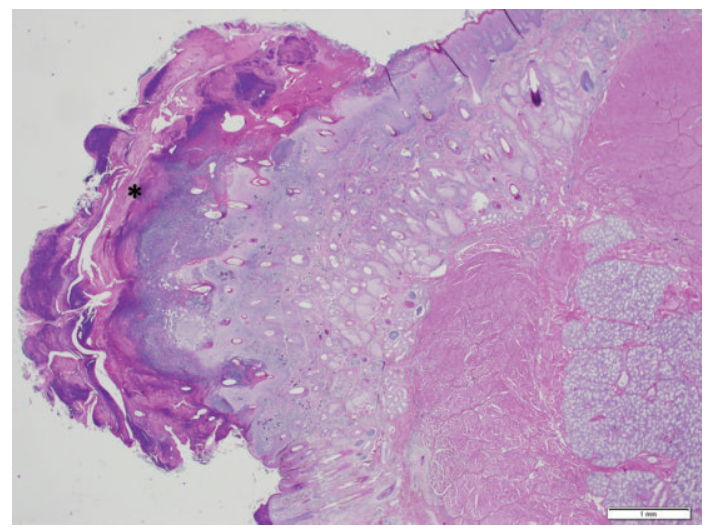

Figure 4: Haired skin, lip commissure. Focally extensive, severe, proliferative, and necrotizing dermatitis, with epithelial hyperplasia, necrosis and loss with replacement by a serocellular crust $(*)$. Hematoxylin and eosin stain, $2 \mathrm{x}$ objective.

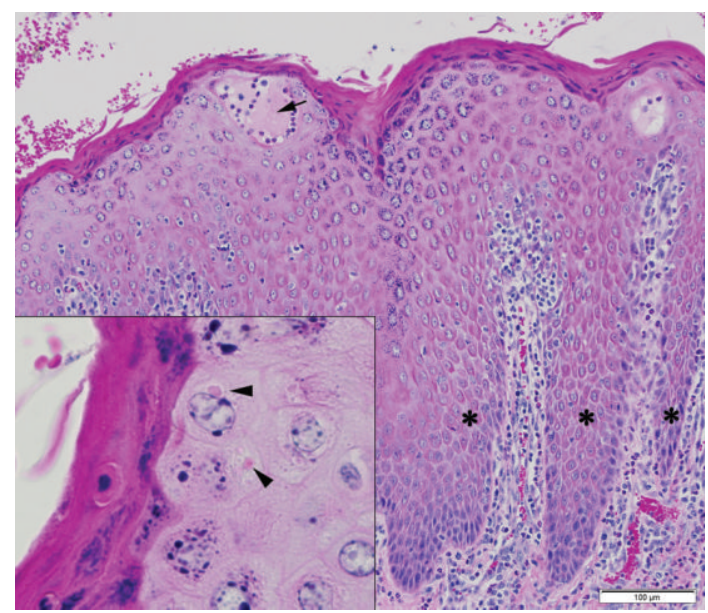

FIgURE 5: Haired skin, lip commissure. Epithelial hyperplasia $(*)$ with increased thickness up to 10 times normal (acanthosis) with prominent intracellular clear spaces/bridging (spongiosis), anastomosing rete ridges, and a vesicle (arrow). Inset: rarely keratinocytes contain one or more $2-10 \mu \mathrm{m}$ round, brightly eosinophilic intracytoplasmic inclusion bodies (arrowheads). Hematoxylin and eosin stain, 20x objective. 


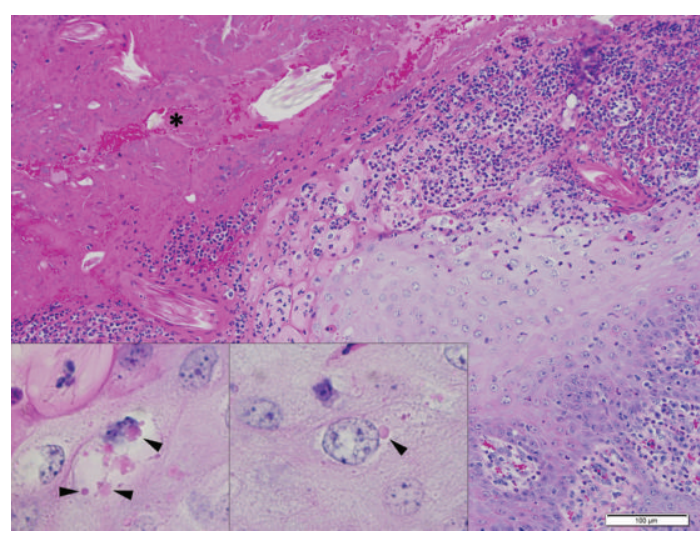

Figure 6: Haired skin, lip commissure. Epithelial hyperplasia, necrosis, and loss with overlying serocellular crust $(*)$ composed of keratin, proteinaceous fluid, degenerate neutrophils, and numerous mixed bacteria. Insets: rarely keratinocytes demonstrate large clear cytoplasmic vacuoles (ballooning degeneration) (left) and contain one or more $2-10 \mu \mathrm{m}$ round, brightly eosinophilic intracytoplasmic inclusion bodies (left and right, arrowheads). Hematoxylin and eosin stain, 20x objective.

replacement by a thick serocellular crust composed of keratin, proteinaceous fluid, degenerate neutrophils, necrotic cellular debris, and numerous mixed bacteria. Within the subjacent dermis, there were numerous dilated small caliber blood vessels separated by edema, fibrin, and moderate numbers of perivascular and perifollicular neutrophils, histiocytes, and lymphocytes.

All tissues for transmission electron microscopy evaluation were fixed in $4 \%$ glutaraldehyde, postfixed in $2 \%$ osmium tetroxide, dehydrated ethanol, and embedded in Epon 812 (Electron Microscopy Sciences, Hatfield, PA, USA). Ultrathin sections $(70-90 \mathrm{~nm})$ were contrasted with lead citrate and uranyl acetate and examined with a Jeol 100CX II electron microscope. Ultrastructurally, the haired skin at the lip revealed degenerate keratinocytes containing numerous typical 200-300 nm wide cytoplasmic parapoxvirus virions (Figure 7).

\section{Discussion}

Contagious ecthyma, also called contagious pustular dermatitis, ecthyma contagiosum, and scabby mouth, is a zoonotic disease, called Orf in humans, with worldwide distribution that affects sheep, goats, wild artiodactyls, and man [2-14]. The causative agent is a double-stranded DNA virus that is a member of the family Poxviridae, subfamily Chordopoxvirinae, genus Parapoxvirus [2, 4, 6-10, 13, 15]. ORFV gains entry through abraded skin and replicates in epidermal cells $[2,4,7-10,13,14]$. Skin lesions progress in an orderly fashion through multiple stages: erythema, macule, papule, vesicle, pustule, scab, and scar [2-7, 9-11, 13, 14]. Infection is confined to the squamous epithelium and may involve the oral cavity, eyelids, teats, and coronary band, subsequently predisposing affected animals to secondary infections [27, 9-11, 13, 14]. Rarely, lesions extend to the squamous

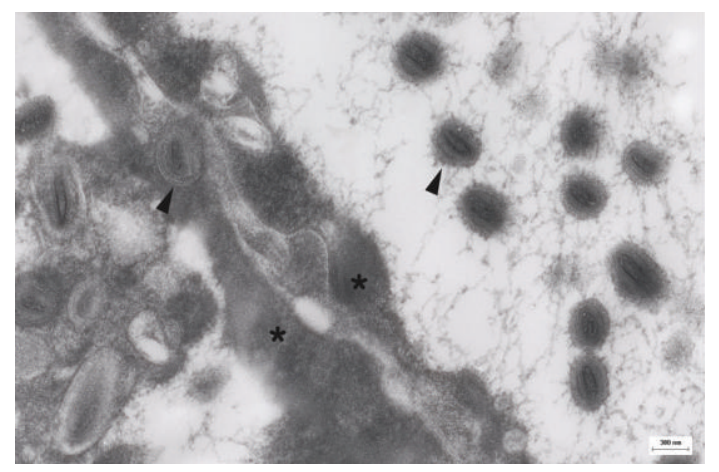

FIgURE 7: Lip. Two keratinocytes with adjacent degenerate cell membranes $(*)$ contain numerous $200-300 \mathrm{~nm}$ brick-shaped virions (arrowheads) free within the cytoplasm. Virions show typical parapoxvirus characteristics of an inner core surrounded by an intermediate coat further bounded by an envelope. Transmission electron microscope, lead citrate and uranyl acetate. Bar $=300 \mathrm{~nm}$.

epithelium of the esophagus, rumen, and omasum, causing ulcerative gastroenteritis $[2,3,5,16]$. Residual skin lesions are not infective once the scab falls off, but substantial amounts of infective virus are shed within scabs which can remain infective in the environment for years $[2,4-9,13]$. The disease has high morbidity and low mortality but can cause significant debilitation due to the inability of affected animals to suckle or graze [5,7-11]. Nursing animals often transfer the virus to adults, typically affecting the teats and udder $[2,4,5,7,8,10-12,14]$. There are several approved commercial vaccines for ORFV which contain virulent virus $[9,11]$. These vaccines are valuable because they limit the severity of disease. The purpose of the vaccine is to produce a lesion at the vaccine site, thus inducing immunity; however, this also causes virus shedding and can contribute to maintenance of infective virus within the environment. Thus, the ORFV vaccine is only recommended for use in endemically infected herds $[9,11]$.

Gross lesions are characterized by multifocal to coalescing proliferative and ulcerative dermatitis which is localized to the squamous epithelium at mucocutaneous junctions, particularly around the mouth and nares [2, 4-6, 10, 12]. Histologic lesions are characterized by marked epidermal hyperplasia, ballooning degeneration, and eosinophilic intracytoplasmic inclusion bodies within keratinocytes that are only briefly detectable at the vesicular stage $[2,4,5,9,10,14]$. There are frequently superimposed bacterial infections in the affected skin [11]. Ultrastructurally, the cytoplasmic inclusions contain numerous 200-300 nm wide brick-shaped virions typical of parapoxviruses with the characteristic inner core surrounded by an intermediate coat further bounded by an envelope [17].

Sheep previously exposed to ORFV can be repeatedly infected, although the severity of the lesions and time to resolution diminish with each subsequent infection $[2,7,9$, 11]. This indicates that the host immune response can control the severity of disease but cannot prevent reinfection [7]. The reason is partially due to the presence of immunomodulatory proteins expressed by ORFV that suppress elements of the 
host immune and inflammatory response $[3,6-9,18]$. These immunomodulatory proteins produce a variety of effects, such as protecting the virus from the antiviral effects of interferons, thus allowing viral replication, or inhibiting the biological activity of important immune system cytokines and suppressing inflammation $[3,6-9,13,18]$.

\section{Conclusions}

According to the limited information from the vendor, this animal originated from a closed flock on site and was subject to the facility's preventive medicine management plan. All sheep receive daily health checks for any abnormalities. Lambs were born on site receive vaccines (BarVac CD/T, Boehringer Ingelheim Vetmedica, St. Joseph, MO, USA, and Scabivax Forte, MSD Animal Health, Summit, NJ, USA) and are dewormed for intestinal parasites. All animals at this vendor remain at the facility their entire life until being sold or used on a protocol.

According to the ORFV vaccine manufacturer's product data sheet, a successful vaccination known as-a "take"results in vaccine site (axilla or behind the elbow in ewes and lambs) erythema 1-14 days after vaccination, with vesicles and pustules approximately 3-14 days after vaccination. Rupture of the vesicles and pustules, with subsequent scab formation, can be expected from about 7 days after vaccination [19]. The time of exposure to ORFV in the environment and the appearance of clinical signs are about 4 to 14 days, which is similar to that of ORFV vaccine clinical signs [2].

This vaccine is similar to the smallpox vaccine in humans, whereby a live virus (vaccinia virus) is inoculated and a "take" is determined to be a successful correlate of immunity. Generalized vaccinia and progressive vaccinia following vaccination by this live vaccine are among the recognized adverse reactions in immunosuppressed humans [20-23]. They result from blood-borne dissemination of the virus, and lesions emerge 6-9 days after vaccination [20-23]. Although the exact timing of vaccination at the vendor's facility could not be determined in this case, based on this animal's history, clinical signs, histopathological evaluation, and electron microscopy results, we believe that this animal developed a clinical case of ORFV either due to an adverse reaction from the ORFV vaccine, or this animal simply had a case of preexisting ORFV, the severity of which may or may not have been affected by vaccination at the vendor's facility. Contributing factors to either scenario are the stress of transport and new environmental surroundings that likely resulted in immunosuppression of this animal with subsequent ORFV disease manifestations.

Standard procedures taken by a facility after a positive ORFV case is identified should include euthanasia of all animals in direct contact with the positive case, as well as decontamination of the premises. In this case, this animal's penmate was euthanized. Within our facility, all animal rooms are routinely cleaned and disinfected between animal shipments with Sani-Plex 128, a quaternary ammonium chloride (Quip Labs, Wilmington, DE, USA) and Chlordet, a potassium hydroxide mixture (Quip Labs, Wilmington, DE, USA) by trained personnel wearing proper personal protective equipment (PPE). All removable husbandry and enrichment items are autoclaved. All uneaten food is discarded. All direct contact with animals is conducted by trained personnel wearing disposable PPE consisting of water resistant coveralls, shoe covers, hair bonnet, mask, and gloves.

Research facilities that work with ORFV-susceptible species that will potentially become immunosuppressed, stressed, or undergo significant surgical alteration as part of experimental protocols should consider using animals originating for ORFV-free facilities to prevent potential complications associated with this organism since substantial amounts of infective ORFV are shed within scabs that can remain infective in the environment for years $[2,4-9,13]$.

\section{Abbreviations \\ ORFV: Orf virus \\ WRAIR/NMRC: Walter Reed Army Institute of Research/Naval Medical Research Center.}

\section{Disclaimer}

Material has been reviewed by the Walter Reed Army Institute of Research. There is no objection to its presentation and/or publication. The opinions or assertions contained herein are the private views of the author, and are not to be construed as official, or as reflecting true views of the Department of the Army or the Department of Defense. The authors declare no competing interests. Research was conducted in compliance with the Animal Welfare Act and other federal statutes and regulations relating to animals and experiments involving animals and adheres to principles stated in the Guide for the Care and Use of Laboratory Animals, 8th edition [1].

\section{Conflict of Interests}

The authors declare no potential conflict of interests with respect to the research, authorship, and/or publication of this paper.

\section{Acknowledgments}

The authors wish to thank the following individuals from the WRAIR/NMRC Veterinary Services Program: Ms. Marcia Caputo, for animal husbandry and health records; $\mathrm{Mr}$. Michael Proctor, for histologic preparations; Mr. Edward Asafo-Adjei, for electron microscopy; Mr. Bartholomew Taylor and Mr. Matthew Wise, for photography and imaging; and Dr. Jennifer Chapman and Dr. Paul Facemire for technical review.

\section{References}

[1] Institute for Laboratory Animal Research, Guide for the Care and Use of Laboratory Animals, National Academies Press, Washington, DC, USA, 8th edition, 2011. 
[2] D. E. Anderson, D. M. Rings, and D. G. Pugh, "Diseases of the integumentary system," in Sheep and Goat Medicine, D. G. Pugh, Ed., pp. 203-204, W.B. Saunders Company, Philadelphia, Pa, USA, 1st edition, 2002.

[3] D. Deane, C. J. Mcinnes, A. Percival et al., "Orf virus encodes a novel secreted protein inhibitor of granulocyte-macrophage colony-stimulating factor and interleukin-2," Journal of Virology, vol. 74, no. 3, pp. 1313-1320, 2000.

[4] H. B. Gelberg, "Alimentary system and the peritoneum, omentum, mesentery, and peritoneal cavity," in Pathologic Basis of Veterinary Disease, J. F. Zachary and M. D. McGavin, Eds., pp. 326-327, Elsevier, St. Louis, Mo, USA, 5th edition, 2012.

[5] P. E. Ginn, J. E. K. L. Mansell, and P. M. Rakich, "Skin and appendages," in Jubb, Kennedy, and Palmer's Pathology of Domesic Animals, M. G. Maxie, Ed., vol. 1, pp. 664-666, Elsevier, Philadelphia, Pa, USA, 5th edition, 2007.

[6] D. M. Haig, "Subversion and piracy: DNA viruses and immune evasion," Research in Veterinary Science, vol. 70, no. 3, pp. 205219, 2001.

[7] D. M. Haig, "Orf virus infection and host immunity," Current Opinion in Infectious Diseases, vol. 19, no. 2, pp. 127-131, 2006.

[8] D. M. Haig, C. J. Mcinnes, J. Thomson, A. Wood, K. Bunyan, and A. Mercer, "The orf virus OV20.0L gene product is involved in interferon resistance and inhibits an interferon-inducible, double-stranded RNA-dependent kinase," Immunology, vol. 93, no. 3, pp. 335-340, 1998.

[9] D. M. Haig and A. A. Mercer, "Orf," Veterinary Research, vol. 29, no. 3-4, pp. 311-326, 1998.

[10] A. M. Hargis and P. E. Ginn, "The integument," in Pathologic Basis of Veterinary Disease, J. F. Zachary and M. D. McGavin, Eds., p. 1023, Elsevier, St. Louis, Mo, USA, 5th edition, 2012.

[11] F. A. Murphy, E. P. J. Gibbs, M. C. Horzinek, and M. J. Studdert, "Poxviridae," in Veterinary Virology, pp. 289-291, Academic Press, San Diego, Calif, USA, 3rd edition, 1999.

[12] C. B. Navarre, M. Q. Lowder, and D. G. Pugh, "Oral-esophageal diseases," in Sheep and Goat Medicine, D. G. Pugh, Ed., pp. 6667, W.B. Saunders Company, Philadelphia, Pa, USA, 1st edition, 2002.

[13] L. J. Savory, S. A. Stacker, S. B. Fleming, B. E. Niven, and A. A. Mercer, "Viral vascular endothelial growth factor plays a critical role in orf virus infection," Journal of Virology, vol. 74, no. 22, pp. 10699-10706, 2000.

[14] I. R. Tizard, "Regulation of adaptive immunity," in Veterinary Immunology, p. 217, Elsevier, St. Louis, Mo, USA, 9th edition, 2013.

[15] International Committee on Taxonomy for Viruses, "Virus Taxonomy: 2012 release (current)," http://www.ictvonline.org/ virusTaxonomy.asp? version $=2012 \&$ bhcp $=1$.

[16] J. F. Zachary, "Mechanisms of microbial infections," in Pathologic Basis of Veterinary Disease, J. F. Zachary and M. D. McGavin, Eds., p. 210, Elsevier, St. Louis, Mo, USA, 5th edition, 2012.

[17] N. F. Cheville and H. Lehmkuhl, "Cytopathology of viral diseases," in Ultrastructural Pathology: The Comparative Cellular Basis of Disease, pp. 318-327, Wiley-Blackwell, Danvers, Mass, USA, 2nd edition, 2009.

[18] Z. Lateef, S. Fleming, G. Halliday, L. Faulkner, A. Mercer, and M. Baird, "Orf virus-encoded interleukin-10 inhibits maturation, antigen presentation and migration of murine dendritic cells," Journal of General Virology, vol. 84, no. 5, pp. 1101-1109, 2003.
[19] MSD Animal Health United Kingdom, "Scabivax Forte Data Sheet," 2012, http://www.msd-animal-health.co.uk/products_ public/scabivax_forte/product_data_sheet.aspx.

[20] J. J. Esposito and F. Fenner, "Poxviruses," in Virology, pp. 28852916, Lippincott Williams and Wilkins, Philadelphia, Pa, USA, 4th edition, 2001.

[21] D. A. Henderson, T. V. Inglesby, J. G. Bartlett et al., "Smallpox as a biological weapon: medical and public health management," Journal of the American Medical Association, vol. 281, no. 22, pp. 2127-2137, 1999.

[22] D. J. McClain, "Smallpox," in Textbook of Military Medicine, pp. 539-559, Office of the Surgeon General, Borden Institute, Washington, DC, USA, 1997.

[23] J. G. Breman and D. A. Henderson, "Diagnosis and management of smallpox," New England Journal of Medicine, vol. 346, no. 17, pp. 1300-1308, 2002. 

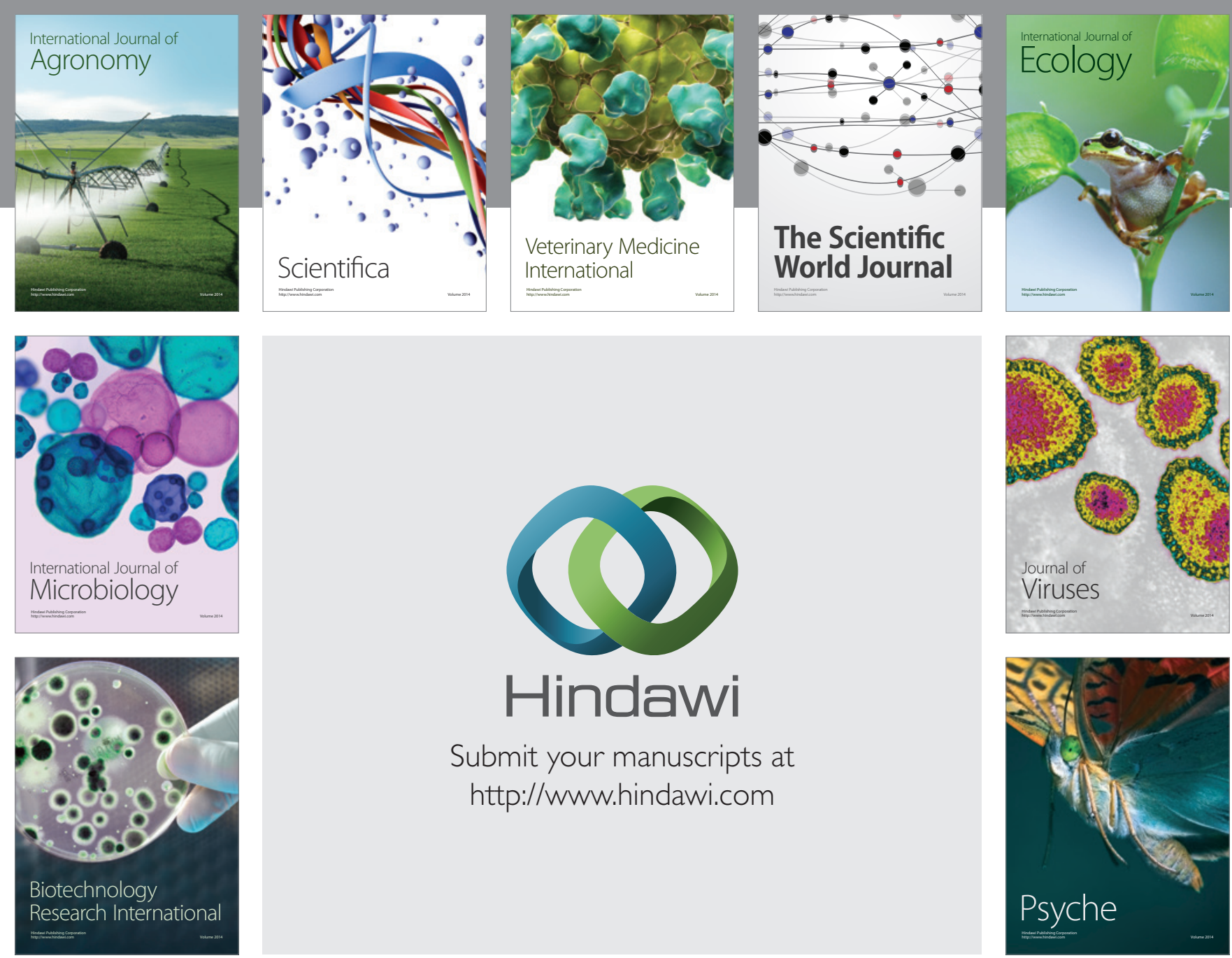

Submit your manuscripts at http://www.hindawi.com
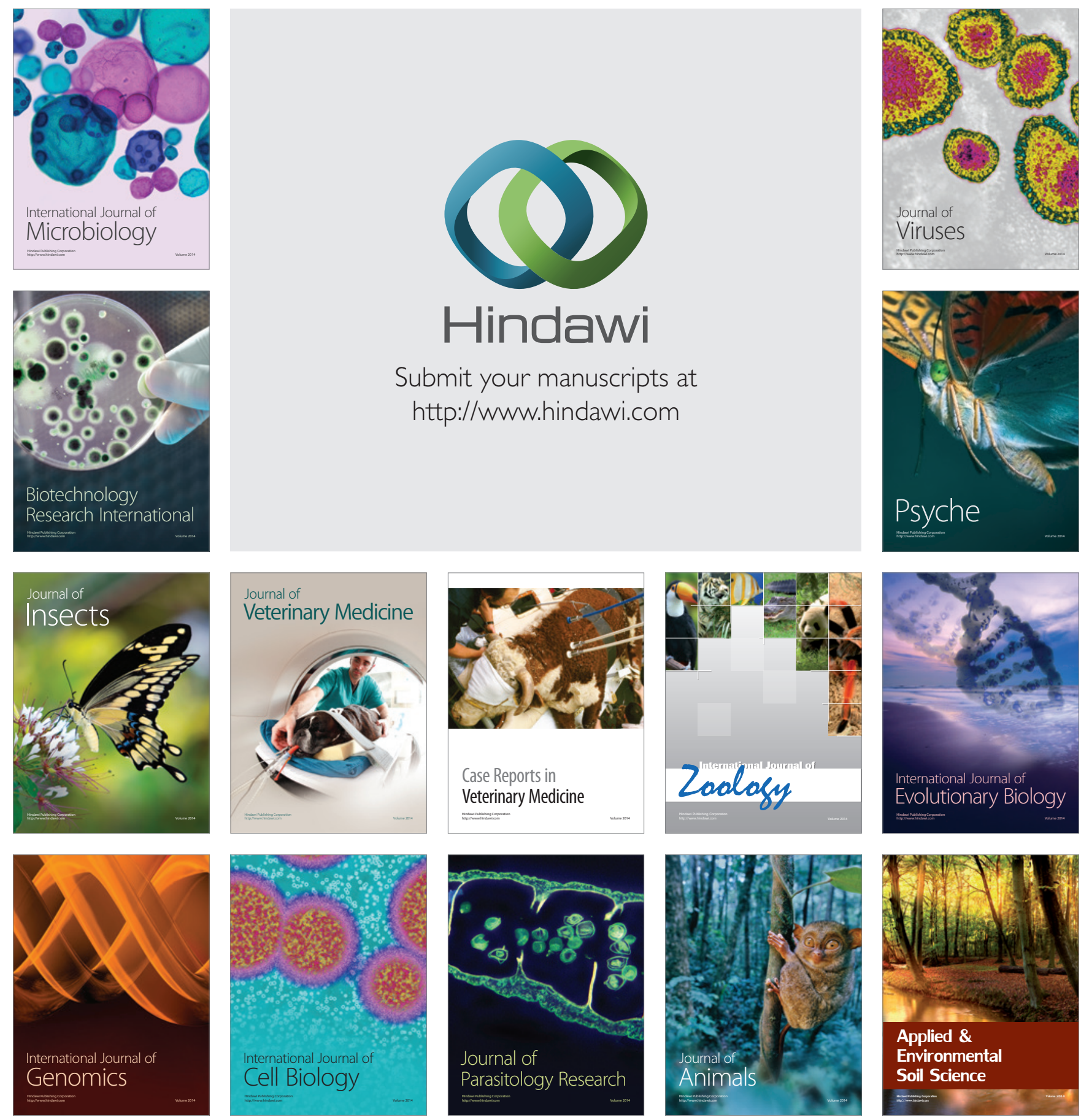